\title{
Aspectos clínicos e radiográficos de osteodistrofias de origem nutricional no cão jovem
}

\author{
Clinical and radiographic aspects of nutritional \\ osteodystrophies in dogs
}

\author{
Sonia da Silva CROCHIK ${ }^{1}$; Masao IWASAKI'; Franklin de Almeida STERMAN ${ }^{1}$; \\ Flávio PRADA ${ }^{2}$
}

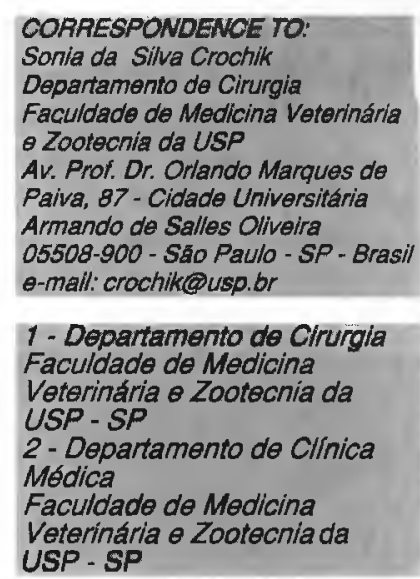

\section{RESUMO}

Foram analisadas radiografias de 634 animais de espécie canina, jovens, de ambos os sexos e raças variadas, encaminhados ao Serviço de Radiologia do Hospital Veterinário da Faculdade de Medicina Veterinária e Zootecnia da Universidade de São Paulo, no período de janeiro de 1982 a dezembro de 1991, com suspeitas clínicas de osteopatia metabólica. A interpretação dos exames radiográficos possibilitou o diagnóstico de Hiperparatireoidismo Secundário Nutricional, Raquitismo ou Osteodistrofia Hipertrófica. O Hiperparatireoidismo Secundário Nutricional foi a patologia mais freqüente das 3; entre os cães de raças puras acometeu mais aqueles de raças grandes e gigantes e também foi mais freqüente no sexo masculino. O mesmo observamos com a Osteodistrofia Hipertrófica, que também foi mais freqüente em machos e, entre as raças puras, nas grandes e gigantes. Apenas 11 casos de Raquitismo foram diagnosticados no período de estudo.

UNITERMOS: Radiografia; Cāes; Osteodistrofia; Nutrição.

\section{INTRODUÇÃO}

$\mathrm{O}$ conhecimento de que o cão é uma animal carnívoro faz com que esta espécie muitas vezes seja erradamente alimentada com dietas exclusivamente à base de carne ou, pior ainda, com as tāo bem conhecidas "sobras da mesa", deixando-se de lado componentes importantes que originalmente supriam as necessidades desta espécie na natureza. Por outro lado, a impressão muitas vezes falsa de que cães de raças conhecidas como grandes e gigantes devem receber alimentação e suplementaçōes em grande quantidade também acaba por prejudicar o desenvolvimento normal desses animais. Por isso, doenças do sistema ósseo resultantes de dietas desequilibradas são comuns em cães em crescimento. Algumas delas apresentam aspectos clínicos semelhantes, tornando-se de fundamental importância o exame radiográfico para diferenciálas e assim estabelecer-se a terapêutica mais adequada. Este trabalho tem como objetivo realçar a importância do conhecimento clínico e radiográfico de algumas doenças ósseas metabólicas de origem nutricional e de fornecer dados a respeito de sua distribuição racial, etária e sexual, na população atendida pelo Hospital Veterinário da Faculdade de Medicina
Veterinária e Zootecnia da Universidade de São Paulo, no período de 1982 a 1991.

\section{LITERATURA}

O cálcio, além de componente estrutural essencial para o esqueleto, está envolvido em muitos processos orgânicos como contração muscular, coagulação de sangue, e liberação de hormônios. Assim, os níveis de cálcio circulante devem ser bem controlados. A concentração de cálcio no plasma é regulada por hormônios calciotrópicos por meio de sistemas que envolvem o intestino, os rins e o osso ${ }^{6}$. Os hormônios calciotrópicos que incluem o paratormônio (PTH), a calcitonina (CT) e os metabólitos da vitamina D têm em comum a habilidade de regular o metabolismo do cálcio com influência direta na mineralizaçāo do esqueleto. PTH e CT são sintetizados nas glândulas paratireóides e tireóides respectivamente, enquanto os metabólitos da vitamina $D$, no cão, existem como formas hidroxiladas da vitamina $D$ que provém da dieta. Os hormônios calciotrópicos regulam o metabolismo do cálcio em um esforço de manter a concentração de cálcio circulante dentro de limites estreitos. Como o cálcio tem de ser absorvido da dieta, estes hormônios 
calciotrópicos e conseqüentemente o crescimento do esqueleto no animal jovem são influenciados pela nutrição ${ }^{7}$. Em cães em crescimento, além de uma grande variedade de doenças ósseas congênitas e de desenvolvimento, ressaltam-se, em nosso meio, aquelas de origem nutricional. Entre elas, 0 de Hiperparatireoidismo Secundário Nutricional é destacado como o de maior importância.

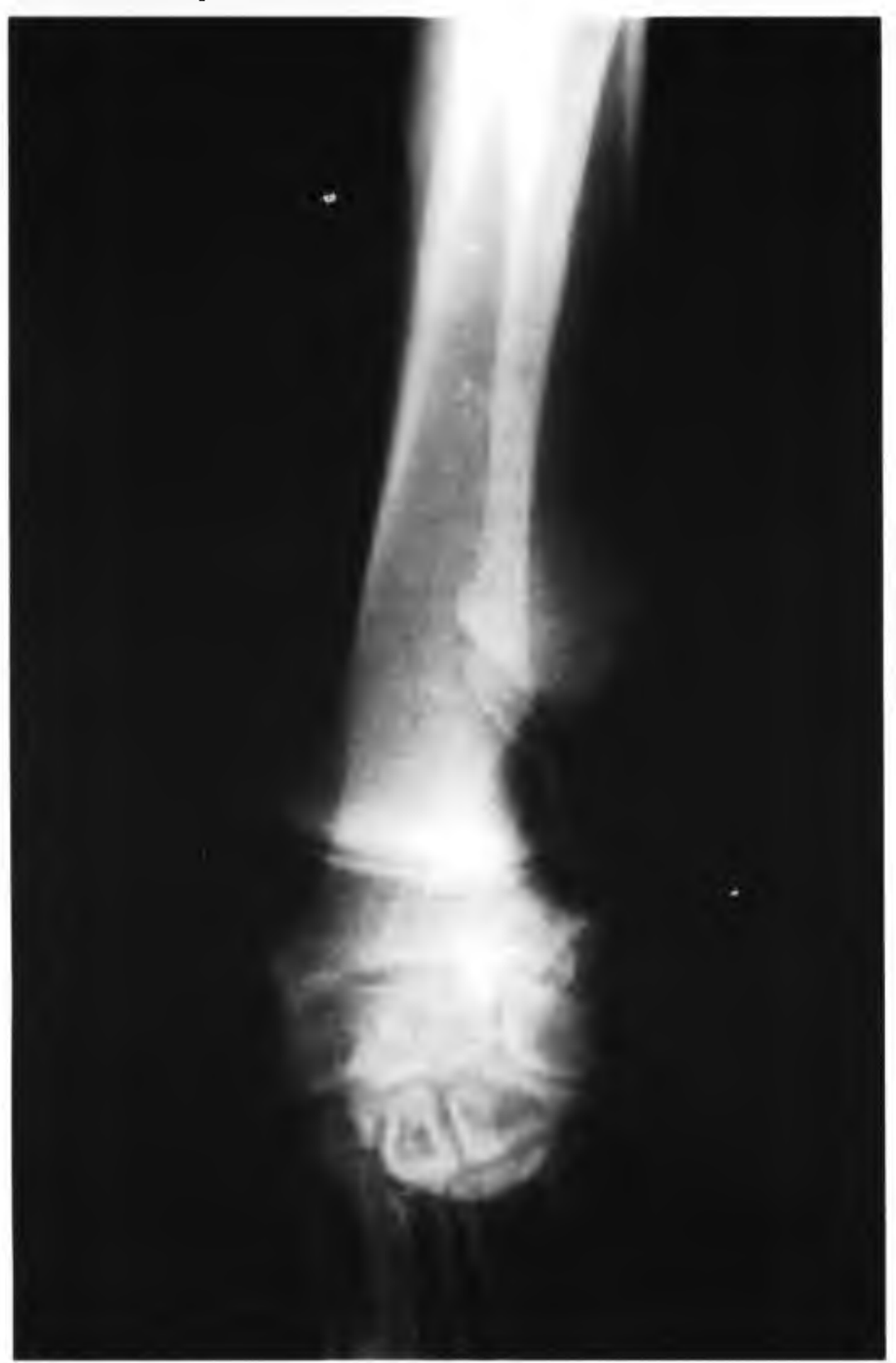

Figura 1

Hlperparatireoldismo Secundárlo Nutrlclonal: Radiografia dorso-palmar de regiáo dlstal de rádlo e ulna demonstrando osteopenia, adelgaçamento de cortlcals em aláfises e densidade elevada em metáflses de rádlo e ulna.

O Hiperparatireoidismo Secundário Nutricional é uma das doenças nutricionais mais freqüentes em cães jovens ${ }^{3}$. Desenvolve-se em cães jovens quando alimentados com dietas pobres em cálcio e/ou ricas em fósforo. O conteúdo de cálcio nos alimentos é bastante variado. Ossos, derivados do leite e plantas leguminosas contêm grandes quantidades de cálcio, enquanto a maioria dos cereais, a carne e outros tecidos animais contêm pequenas quantidades. Muitos alimentos que possuem quantidades significativas de cálcio também apresentam grandes quantidades de fósforo ${ }^{10}$. O coração e fígado bovinos, por exemplo, apresentam valores baixos de cálcio e uma relação cálcio/fósforo de $1: 20$ até $1: 50^{4,5}$. Importante observar que a relação entre os dois elementos cálcio e fósforo - deve-se manter aproximadamente 1,2:1 a $1,4: 1$ na espécie canina ${ }^{14}$. A hipocalcemia é estímulo para a hiperatividade da paratireóide. Esta pode ser induzida, do ponto de vista nutricional, por uma dieta pobre em cálcio, rica em fósforo, ou ambas as situações. Em resposta à hipocalcemia de origem nutricional, as células da

paratireóide sofrem hipertrofia e hiperplasia. O excesso de paratormônio impede a mineralização do osteóide.

As alterações radiográficas observadas são mineralização inadequada dos ossos - que parecem ter quase a mesma densidade dos tecidos-moles -; crescimento normal dos discos epifisários; corticais extremamente delgadas; pode estar presente uma linha de maior densidade nos bordos epifisários das metáfises (Fig. 1) fraturas patológicas em ossos longos e em vértebras torácicas e lombares ${ }^{11}$. O aparecimento dos sintomas se dá, em geral, entre 3 a 5 meses de idade ${ }^{y}$. Animais severamente afetados podem apresentar paresia ou paralisia resultante de fraturas em ossos longos e compressão de vértebras, constipação como resultado da diminuição dos diâmetros da pelve e até convulsões ${ }^{3,15}$.

O Raquitismo é reconhecido como doença tanto no homem quanto nos animais desde 1650 . O cão é capaz de sintetizar vitamina $\mathrm{D}$ na pele através de compostos lipídicos sob a influência de raios ultravioleta. Porém, esta quantidade parece não ser suficiente para as suas necessidades diárias . Deficiências, entretanto, são raras e níveis críticos são atingidos apenas quando os níveis de cálcio e fósforo estão baixos. A combinação desses acontecimentos levaria ao aparecimento do Raquitismo ${ }^{y}$. A deficiência de vitamina D causa a produção de uma matriz de cartilagem altamente estável e, portanto, não calcificável e difícil de ser reabsorvida. Nos animais jovens, isto resulta na não degeneração de células da cartilagem e os capilares das metáfises são incapazes de penetrar na cartilagem. Há um acúmulo de células de cartilagem que produzem mais matriz não reabsorvível. $O$ disco epifisário conseqüentemente torna-se espessado e irregular $^{3}$. Os sintomas aparecem entre 6 e 14 semanas de idade. Os principais sinais clínicos são retardo do crescimento. apatia, fraqueza muscular e encurvamento dos ossos sob o peso de andar e o estresse da contraçāo muscular. Aumentos nodulares macroscópicos são visíveis nas extremidades dos ossos longos (resultantes dos discos epifisários). São particularmente observados nas regiões distais de rádio e ulna e nas articulações costo-condrais ${ }^{16}$.

As alterações radiográficas do Raquitismo no cão são alargamento dos discos epifisários; falha na mineralização adequada dos ossos; encurvamento dos ossos longos e engrossamento extremo das metáfises adjacentes aos discos epifisários principalmente em rádio e ulna distais e junções costo-condrais; bordos epifisários e metafisários dos discos 
epifisários apresentam-se largos, encurvados e irregulares; diminuição de densidade das metáfises por falha do osteóide metafisário em se calcificar ${ }^{11.16}$ (Fig. 2).

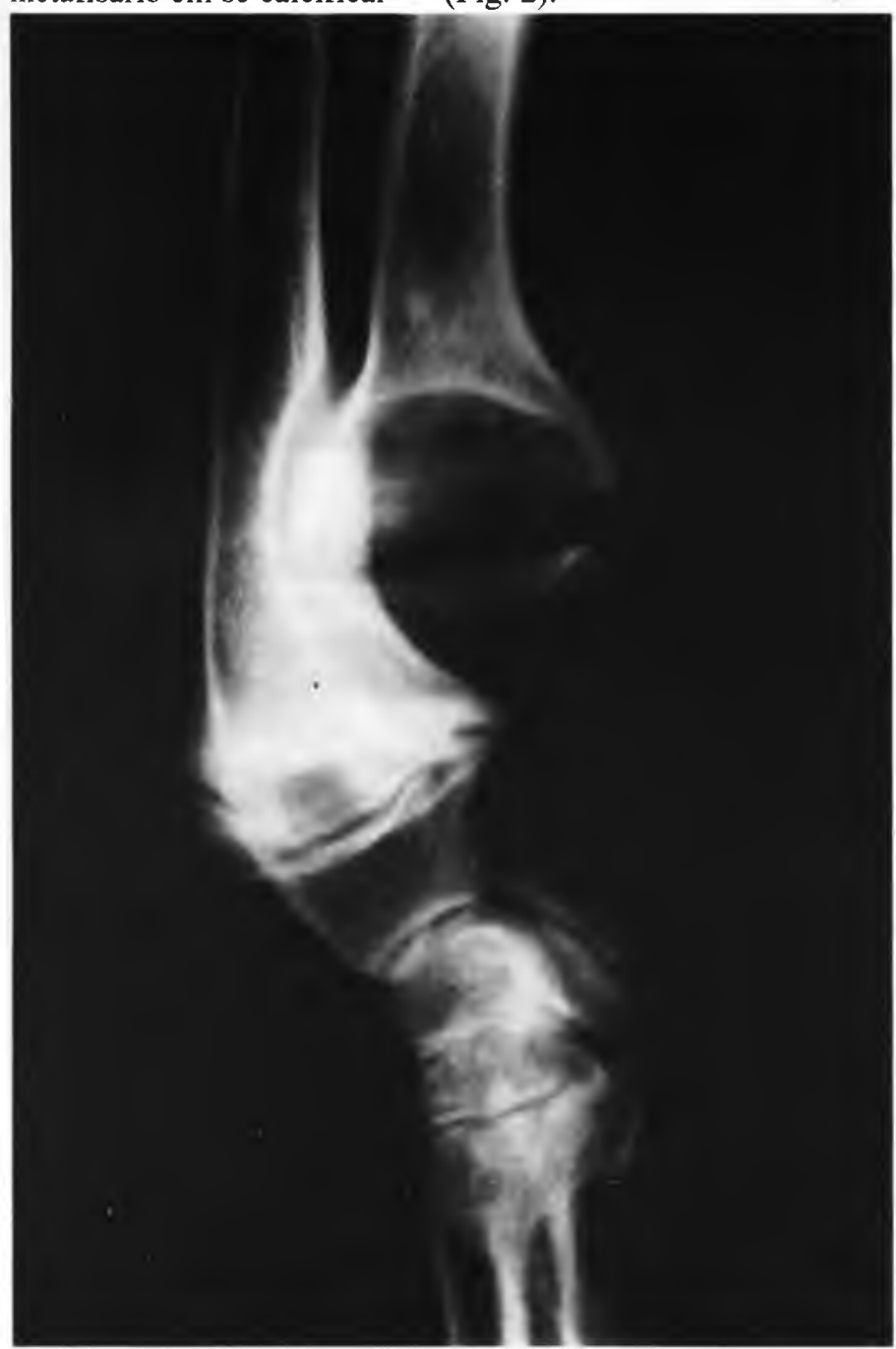

Figura 2

Raquitismo: Radiografia látero-medial de região distal de rádio e ulna demonstrando osteopenia, discos epifisários com diâmetros aumentados, metáfises em forma de cogumelo.

O Osteodistrofia Hipertrófica foi identificada pela primeira vez na literatura veterinária na década de 30 . Afeta principalmente cāes jovens de raças grandes e gigantes, com desenvolvimento rápido ${ }^{12}$. Sua etiologia permanece ainda em discussão. Os sintomas clínicos são variáveis. Geralmente há aumento de volume e sensibilidade das regiões metafisárias dos ossos afetados. A doença pode ser acompanhada de hipertermia variável. Muitos animais apresentam apatia, anorexia e dificuldade em se manter em estação ou em se locomover'. Níveis séricos baixos de vitamina $C$ foram encontrados em cães afetados pela doença ${ }^{13}$. Entretanto, os próprios autores ${ }^{13}$ ressaltam que vitamina $C$ administrada por via intramuscular ou oral nāo só falhou em elevar os níveis séricos, como também não proporcionou qualquer melhora no quadro dos cães. Sabe-se que a "superalimentação" exerce papel importante na reprodução desta e de outras doenças ósseas metabólicas no cão ${ }^{8}$. Dietas com excesso de proteína e energia causam produçāo excessiva de osso e o excesso de cálcio provoca hipercalcitoninismo, o que retarda a reabsorção óssea ${ }^{18}$. O ácido ascórbico também retarda a reabsorção óssea ${ }^{1}$. Já se observaram sinais de processo inflamatório supurativo ao exame histológico das metáfises dos ossos de cães afetados ${ }^{19}$; além disto, também há relato de crescimento de Escherichia coli a partir de cultura de amostra de sangue de um cāo que apresentava os sinais clínicos e radiográficos desta patologia ${ }^{17}$.

Os sinais radiográficos se iniciam com uma linha radiotransparente nas metáfises dos ossos longos; um colar de osso se forma, a seguir, ao redor das metáfises externamente à córtex; as metáfises se tornam mais densas e perdem o padrão trabecular (Fig. 3). Com a progressāo da doença, o colar ósseo se funde, dando a impressão de alargamento da metáfise. Ocasionalmente mandíbula, costelas e escápula podem ser afetadas.

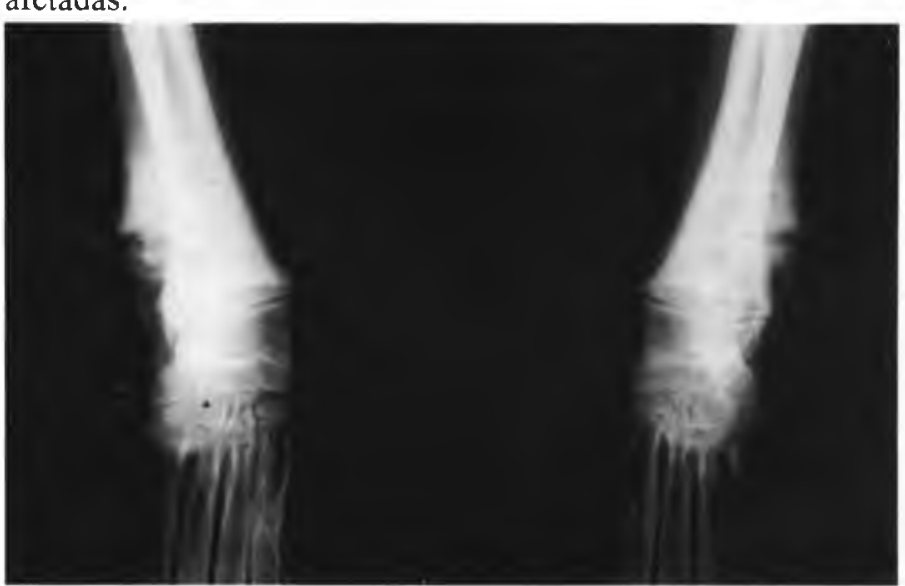

Figura 3

Osteodistrofia Hipentrófica: Radiografia dorso-palmar de regiāo distal de rádio e ulna. Discos epifisários de diâmetros normais, linhas radiotransparentes e discreta reação periostal em metáfises distais de rádio e ulna.

Os sinais clínicos e radiográficos são semelhantes à hipovitaminose C humana (escorbuto infantil). Porém, a Osteodistrofia Hipertrófica é caracterizada por aposição óssea aumentada e diminuição da reabsorção do osso. O escorbuto se caracteriza por osteoporose, ou seja, pouca massa óssea por insuficiência osteoblástica. As duas entidades seriam, portanto, morfológica e patogenicamente diferentes.

\section{MATERIAL E MÉTODO}

Animais: Foram analisados dados de resenha de 634 cães jovens, de ambos os sexos, e de raças diversas, com suspeitas de osteopatias metabólicas, e que tiveram seus diagnósticos definidos por meio de exames clínico e radiográfico. "Os animais foram atendidos no Hospital Veterinário da Faculdade de Medicina Veterinária e Zootecnia da Universidade de São 
CROCHIK, S.S.; IWASAKI, M.; STERMAN, F.A.; PRADA, F. Aspectos clínicos e radiográficos de osteodistrofias de origem nutricional no cão jovem. Braz. J. vet. Res. anim. Sci. São Paulo, v.33, n.4, p.239-243, 1996.

Paulo, no período de janeiro de 1982 a dezembro de 1991.

Técnica radiográfica: Foram realizados exames radiográficos das regiōes distais de crescimento de rádio e ulna. As projeções látero-medial, médio-lateral e dorso-palmar foram as eleitas. As técnicas radiográficas basearam-se em método que relaciona a quilovoltagem e a miliamperagem-segundo com a espessura da região a ser radiografada.

Análise das radiografias: $\mathrm{A}$ análise das radiografias baseou-se na observação de alterações ósseas quanto à densidade, estrutura e morfologia principalmente das zonas de crescimento distais de rádio e ulna.

Análise estatística: Os resultados obtidos foram compilados em tabelas de freqüências com respectivas porcentagens. Quando necessário, foi empregado o teste de duas proporçōes no nível de $5 \%$ de significância $(\mathrm{p}<0,05)^{2}$.

\section{RESULTADOS}

Dos 634 animais cujas radiografias foram por nós analisadas, encontramos $553 \quad(87,22 \%)$ com Hiperparatireoidismo Secundário Nutricional (HPT); 11 (1,73\%) com Raquitismo (RAQ) e 70 (11,04\%) com Osteodistrofia Hipertrófica (ODH).

Os animais apresentando HPT foram representados por 173 $(31,28 \%)$ cães sem raça definida (SDR), cujos portes são variados. Dos $380(68,72 \%)$ cães de raças puras, apenas 15 $(3,95 \%)$ representavam raças de médio ou pequeno portes (Cocker Spaniel 6, Poodle 4, Fox 3, Fox Paulistinha 1, Schnauzer miniatura 1); enquanto $365(96,05 \%)$ eram de raças grandes ou gigantes (Pastor Alemão 178, Doberman 65, Fila Brasileiro 41, Dogue Alemão 21, Collie 14, Boxer 10, Afghan Hound 06, Dálmata 05, Weimaraner 05, Akita 04, Rottweiler 04. Husky Siberiano 02, Mastiff 02, Old English Sheep Dog 02, Setter Irlandês 02, Pointer Inglês 01, São Bernardo 01, Terra Nova 01. Dos 553 animais, $336(60,76 \%)$ eram machos e $217(39,24 \%)$, fêmeas.

Os cães apresentando RAQ foram 04 SRD $(36,36 \%), 03$ da raça Doberman (27,27\%), 02 Afghan Hound (18,18\%) e 02 Pastor Alemão (18,18\%). Do total, $05(45,45 \%)$ eram machos e $06(54,55 \%)$ fêmeas.

A ODH encontrada em 70 cães mostrou-se presente em 3 SRD e 67 cães de raças puras de porte grande ou gigante (Dogue Alemão 18; Pastor Alemão 14, Boxer 13, Doberman 06, Fila Brasileiro 06, Weimaraner 04, Setter Irlandês 03, Collie 02, Mastin Napolitano 01). No que diz respeito ao sexo, $49(70 \%)$, eram machos e $21(30 \%)$ fêmeas.

\section{DISCUSSÃO}

Os mecanismos que mantêm os níveis de cálcio no sangue dependem de hormônios chamados calciotrópicos (paratormônio, calcitonina e metabólitos da vitamina $\mathrm{D}$ ). O cálcio absorvido provém da dieta, portanto podemos supor que todos os sistemas que dele dependem, inclusive o sistema ósseo, são de certa forma influenciados pela nutrição. Entre as patologias ósseas de origem nutricional, chama a atenção o Hiperparatireoidismo Secundário Nutricional por ter sido durante muito tempo a doença nutricional mais comum em cães em muitos países do mundo e talvez ainda seja a mais frequiente em nosso meio, já que esses animais em crescimento freqüentemente recebem dietas não comercializadas e pouco balanceadas. Ela foi a mais freqüente também entre as patologias por nós pesquisadas. As alterações radiográficas observadas por vários autores concordam também com nossas observações. A média de idade dos cães cujas radiografias analisamos foi de 3,58 meses, dentro da faixa etária de aparecimento dos sintomas - entre 3 e 5 meses. Apesar de não haver citação de predisposição racial em cães para esta condição óssea, em nosso estudo a distribuição de raças puras apresentou freqüência muito mais elevada entre aquelas consideradas grandes e gigantes do que entre as consideradas de pequeno e médio porte. O mesmo aconteceu com a distribuição entre os sexos, havendo diferença estatística significativa $(\mathrm{p}<0,05)$ em favor dos machos com Hiperparatireoidismo Secundário Nutricional.

O Raquitismo é resultado de concentrações inadequadas de vitamina $\mathrm{D}$ e fósforo, ou ambos, no sangue. Dietas deficientes, alterações da absorção de fósforo e falha no metabolismo normal da vitamina $\mathrm{D}$ e do fósforo são as causas mais comuns. A vitamina $D$, além de influenciar na reabsorção intestinal e renal de cálcio, participa no processo de mineralizaçāo da cartilagem, para a formação do osso. Como revela a literatura mundial, é condição rara também em nosso meio, tendo sido observados apenas 11 casos no período de 10 anos pesquisados. Além dos quatro cães sem raça definida, a distribuição entre as raças puras revela presença apenas daquelas consideradas grandes. Em nosso estudo, os animais foram radiografados com média de idade de 3,18 meses, concordando também com a literatura.

- O diagnóstico da Osteodistrofia Hipertrófica é importante principalmente para que se possa diferenciá-la de outras patologias que acometem o sistema ósseo e cujos sintomas clínicos se assemelham a ela em alguns aspectos, como é o caso do Raquitismo e do Hiperparatireoidismo Secundário Nutricional. Observamos três cães de raça nāo definida, enquanto os outros 67 casos observados são de cães de raças grandes e gigantes, havendo diferença estatística significativa ( $\mathrm{p}<0,05)$ entre machos (49 casos) e fêmeas (21 casos). Os animais por nós observados tinham 4,27 meses, em média, quando foram radiografados, concordando com a idade do aparecimento dos sintomas citada na literatura. Apesar de ainda não estar estabelecida, é provável que a etiologia da Osteodistrofia Hipertrófica seja multifatorial. Além de prováveis associações com agentes infecciosos, alguns estudos comprovam que a suplementação excessiva de minerais, proteínas e vitaminas agrava ou até mesmo reproduz o processo. 


\section{SUMMARY}

We analysed radiographies of 634 young dogs, males and females, of different breeds, attended at the Veterinary Hospital of the Faculty of Veterinary Medicine and Zootechny of University of São Paulo, from January 1982 to December 1991. The clinical findings were those of metabolic osteopathy. The film readings provided the diagnosis of Nutritional Secondary Hyperparathireoidism, Rickets and Hypertrophic Osteodystrophy. Nutritional Secondary Hyperparathireoidism was the most frequent of the three pathologies. It occurred more in males, and among the purebred dogs it was more frequent in large and giant breeds. The same happened to those whose diagnoses was Hypertrophic Osteodystrophy. Only 11 cases of Rickets were diagnosed during the period of this study.

UNITERMS: Radiography; Dogs; Osteodystrophy; Nutrition.

\section{REFERÊNCIAS BIBLIOGRÁFICAS}

1-ALEXANDER, J.W. Selected skeletal dysplasias. Veterinary Clinics of North America: Small Animal Practice, v.12, n.21, p.64-70, 1982.

2-ARMITAGE, P.; BERRY, G. Statistical methods in medical research. 2.ed. Oxford, Blackwell Scientific Publications, 1987. Cap. 4, p.93-140: Statistica inference.

3-BENNETT, D. Nutrition and bone disease in the dog and cat. Veterinary Record, v.99, n.13, p.313-20, 1976.

4-CAPEN, C.C. Calcium-regulating hormones and metabolic bone disease. In: NEWTON, C.D.; NUNAMAKER, D.M. Textbook of small animal orthopaedics. Philadelphia, J.B. Lippincott Company, 1985. p.673-722.

5-CAPEN, C.C.; ROSOLT, T.J. Calcium-regulating hormones and diseases of abnormal mineral (calcium, phosphorus, magnesium) metabolism. In: KANEKO, J.J. Clinical biochemistry of domestic animals. 4.ed. San Diego, Academic Press, 1989. p.678-752

6-HAZEWINKEL, H.A.W. Calcium metabolism and skeletal development in dogs In: WALTHAM SYMPOSIUM, 7., Harrogate, 1989.

7-HAZEWINKEL, H.A.W. Nutrition in relation to skeletal growth deformities Journal of Small Animal Practice. v.30, n.12, p.625-30, 1989

8-HEDHAMMAR, A.; WU, F.M.; KROOK, L.: SCHRYVER, H.F.; LAHUNTA, A.; WHALEN, J.P.; KALLFELZ, F.A.; NUNEZ, E.A.; HINTZ, H.F.; SHEFFY, B.E.; RYAN, G.D. Overnutrition and skeletal disease: an experimental study ín growing great dane dogs. Cornell Veterinarian, v.64. p.9-130, 1974. Supplement 5 .

9-HOULTON, J.E.F. Diseases of the bone. In: CHANDLER, E.A.; THOMPSON, D.J.; SUTTON. J.B.; PRICE, C.J. Canine medicine and therapeutics. 3.ed. Oxford, Blackwell Scientific Publications, 1991. p.203-29.

10-KALLFELZ, F.A. Skeletal and neuromuscular diseases. In: LEWIS, L.D. MORRIS JÚNIOR, M.L.; HAND, M.S. Small animal clinical nutrition III 3.ed. Topeka, Mark Morris Associates, 1987. Cap. 12, p.1-15.

11-KEALY, J.K. Diagnostic radiology of the dog and cat. Philadelphia, W.B Saunders, 1979. Cap. 4, p.254-375: Bones and joints

12-LENEHAN, T.M.; FETTER, A.M. Hypertrophic osteodystrophy. In: NEWTON, C.D.; NUNAMAKER, D.M. Textbook of small animal orthopaedics. Phipadelphia, J.B. Lippincott Company, 1985. p.597-601

13-MEIER, H.; CLARK. S.T.; SCHNELLE, G.B.; DONALD, H.W. Hypertrophic osteodystrophy associated with disturbance of vitamin C synthesis in dogs. Journal of the American Veterinary Medical Association, v.130, n.4, p.48391, 1957.

14-NATIONAL RESEARCH COUNCIL. Nutrient requirements of dogs. Washington, DC, National Academy Press, 1985. 79p.
15-RISER. W.H. Juvenile osteoporosis (osteogenesis imperfecta). A calcium deficiency. Journal of the American Veterinary Medical Association, v.139, n.1, p.117-9, 1961

16-RISER, W.H. Radiographic differential diagnosis of skeletal diseases of young dogs. Journal of the American Veterinary Radiology Society, v.5, n.1, p.1527,1964

17-SCHULZ, K.; DAYNE, J.T.; ARONSON, E. Escherichia coli bacteremia associated with hypertrophic osteodystrophy in a dog. Journal of the American Veterinary Medical Association, v.199, n.9, p.1170-3, 1991

18-TEARE, J.A.; LENNART, K.; KALLFELZ, F.A.; HINTZ, H. Ascorbic acid deficiency and hypertrophic osteodystrophy in the dog: a rebuttal. Cornell . Veterinarian, v.69, n.4, p.384-401, 1979.

19-WOODARD, J.C. Canine hyperthophic osteodystrophy - a study of the spontaneous disease in lettermates. Veterinary Pathology, v.19, n.3, p.337-54, 1982. 